\title{
Anatomic Single Bundle Anterior Cruciate Ligament Reconstruction Using Two Cross Pins: Cadaveric Study and Clinical Application
}

\author{
Ayman M. Ebied, Adel Elseedy, Mahmoud Tahoun, Wael El-Kholy \\ Orthopaedic \& Anatomy Departments, Faculty of Medicine, Menoufia University Hospitals, Shebin el Kom, Egypt \\ Email: adelelseedy2010@yahoo.com
}

Received 16 April 2014; revised 30 May 2014; accepted 15 June 2014

Copyright (C) 2014 by authors and Scientific Research Publishing Inc.

This work is licensed under the Creative Commons Attribution International License (CC BY).

http://creativecommons.org/licenses/by/4.0/

(c) (i) Open Access

\section{Abstract}

Background: Anatomic single bundle is a widely accepted technique for anterior cruciate ligament (ACL) reconstruction. The research question: are transverse pins safe to fix the ACL graft on the femoral side in anatomic single bundle ACL reconstruction? Material and Methods: Ten cadaveric femoral dry bones were tested in this study. Thirty $\mathrm{mm}$ long sockets were prepared in the center of the anatomic ACL footprints. Transverse pin guide for soft tissue graft was used to prepare pin sites through the femoral sockets using three different positions. The positions (A) and (B) had a lateral entry point and differ in their inclination in relation to the joint line; while (A) has a $15^{\circ}$ open laterally plane, (B) has a $15^{\circ}$ closed laterally. The last position (C) had a medial entry point for the transverse pins. The relations of the transverse pins to the anatomic landmarks (lateral epicondyle and articular cartilage) in addition to the path of the pins within the femoral sockets were recorded. Results: Pins inserted while the guide in position (A) crossed the center of the femoral sockets and were fully seated within the bone of the distal femur. Distal pins inserted through inclination (B) were found to penetrate the posterior cortex of the distal femur proximal to the lateral femoral condyle. Pins inserted from the medial side (C) achieved central position through the femoral tunnel. The entry point for transverse pins through lateral entry points was very close to the attachment of the lateral collateral ligament at the lateral epicondyle while medial entry point avoided important structures. Conclusion: Transverse pins inserted through the lateral entry point carry the risk of penetrating the posterior cortex of the femur and/or damage the attachment of the lateral collateral ligament. Medial entry point may give a safer approach but needs further adjustments of the guides and clinical trials of the technique.

\section{Keywords}

Anterior Cruciate Ligament, Reconstruction, Cross Pins, Application

How to cite this paper: Ebied, A.M., Elseedy, A., Tahoun, M. and El-Kholy, W. (2014) Anatomic Single Bundle Anterior Cruciate Ligament Reconstruction Using Two Cross Pins: Cadaveric Study and Clinical Application. Open Journal of Orthopedics, 4, 150-156. http://dx.doi.org/10.4236/ojo.2014.46025 


\section{Introduction}

The concept of anatomic anterior cruciate ligament (ACL) reconstruction has been widely accepted by knee surgeons [1]. The anatomic double bundle ACL reconstruction technique may not be achievable in all patients due to technical or anatomic causes; therefore the anatomic single bundle ACL reconstruction is commonly practiced. Preparation of the femoral socket through a low arthroscopic medial portal has been shown to provide better access to the native ACL femoral footprint and consequently an anatomic reconstruction [1] [2].

The bio-absorbable cross pins (Rigid-Fix; Mitek, J\&J) achieves fixation of the graft within the femoral tunnel at two points that is close to the joint line; produce less tunnel widening than cortical suspensory methods [3] and found to provide superior biomechanical stability of the ACL grafts than the interference screws at 6 weeks [4]. However, the cross pin technique has originally been described for graft fixation on the femoral side through the trans-tibial technique.

Recent cadaveric study on the use of transverse pin (ACL system, Stryker) that secures the ACL graft within the femoral tunnel revealed a risk of injuring or being very close to important lateral structures like the lateral collateral ligament (LCL) and popliteus tendon [5].

Doubts have been raised about the safety of using this technique on anatomic single bundle ACL reconstruction [6]. It is not clear whether the femoral Rigid-Fix pins can be safely used for anatomic single bundle ACL reconstruction performed through the medial portal and if there are special precautions that should be considered on using this method of fixation?

This article is presenting laboratory work on cadaveric bones studying the use of Rigid-Fix pins on anatomic single bundle ACL reconstruction, its risks, technical problems and possible solutions.

\section{Material and Methods}

Ten human cadaveric femora were tested in this study (5 were right and 5 left). The femora were fixed in a position to assume a knee flexion angle of $110^{\circ}$ (Figure 1). An anatomic position for the ACL footprint was chosen according to criteria described by Van Eck et al. [7] to create a femoral socket in each specimen. A drill pin was driven through the chosen position and $8 \mathrm{~mm}$ diameter-30 $\mathrm{mm}$ length femoral sockets were prepared using femoral reamers from ACL system (MITEK, DePuy, Johnson \& Johnson).

The Rigid-Fix guide was used to prepare the insertion site for two pins across the femoral sockets. Taking the joint line of the posterior femoral condyles as a reference line (Figure 1); three inclinations for insertion of the Rigid-fix sleeves were chosen: the first inclination (A) started drilling for the pins from the lateral side and created a laterally closed $15^{\circ}$ angle with the reference line i.e. (posterior to anterior inclination of the pins).

The second inclination (B) created a laterally open $15^{\circ}$ angle with the same reference line (Figure 1) (anterior to posterior direction of the pins). In the third inclination (C) the drilling started from the medial side with an

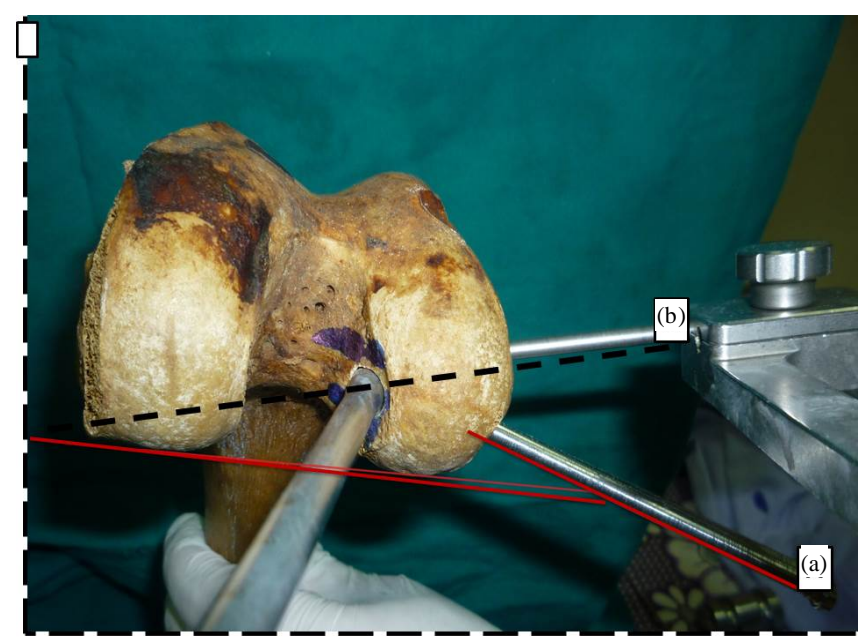

Figure 1. The femur is fixed to assume a position of $110^{\circ}$ knee flexion. The cross pin guide inserted through the prepared femoral tunnel and sleeves inserted from the lateral side through two inclinations: The first (a) and the second (b). 
anterior to posterior inclination while driving the sleeves across the femoral condyles (Figure 2). These inclinations were arbitrarily selected to allow the cross pins to traverse the femoral tunnels and femoral condyle without harming the important structures like the articular cartilage or the attachment of the collateral ligaments.

The positions of the pins in relation to the femoral tunnels were recorded in each experiment as well as the distance between the entry points and the lateral epicondyle for inclinations that started laterally and the medial epicondyle for the medial one.

The averages of these distances were calculated as well as the range and standard deviation.

\section{Results}

Position of the pins in relation to the femoral tunnel:

Inclination A: all pins driven through this inclination achieved central position within the femoral tunnel and tip of the pin stayed within the bone of the distal femur (Figure 3).

Inclination B: the proximal of the two cross pins had achieved a central position inside the femoral tunnels and remained within bone throughout its length. In contrast, the distal pin though passed through the femoral tunnels, its tip penetrated the posterior cortex of the distal femur (Figure 4).

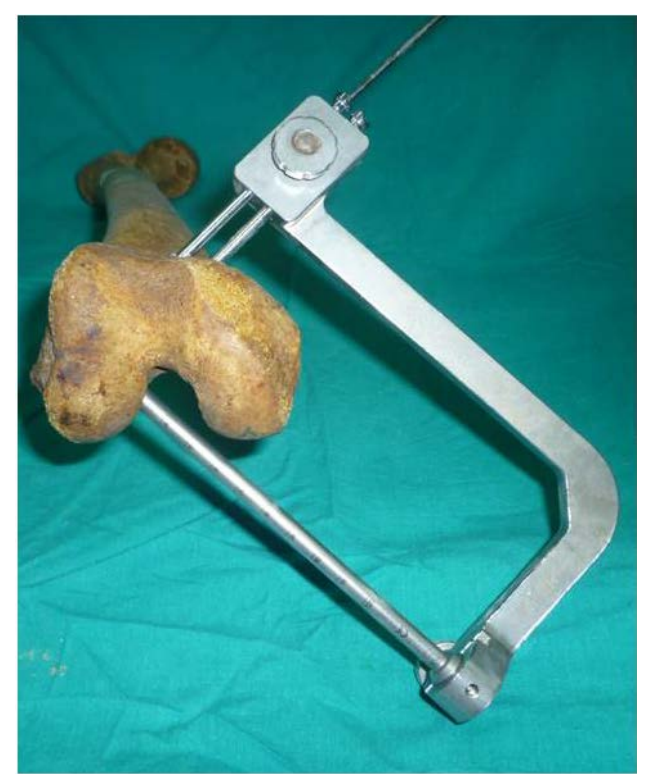

Figure 2. It shows the third inclination C. The cross pin guide was shifted to allow insertion of pins from the medial side. The inclination of the sleeves from anterior to posterior.

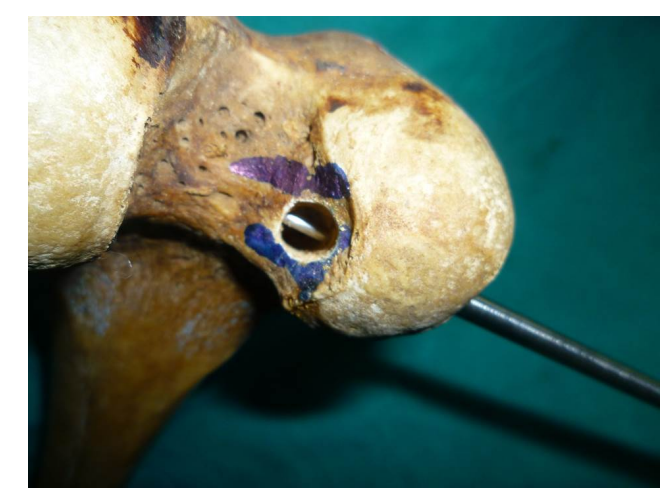

Figure 3. Pins inserted using inclination A achieved central position within the femoral tunnel. 


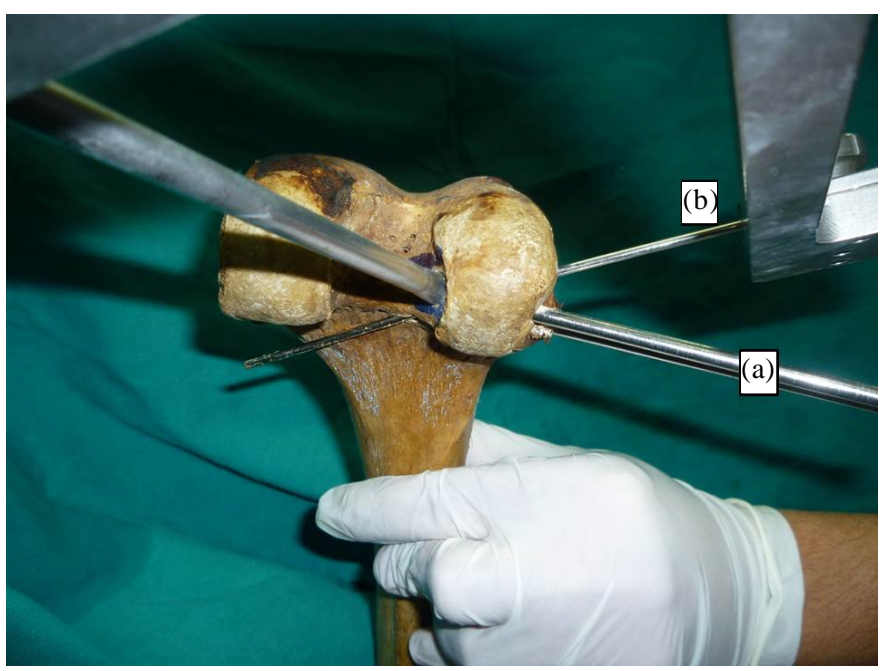

Figure 4. Distal pins inserted through inclination B penetrated the posterior cortex of the femur.

Inclination C: all pins driven through this inclination achieved central position within the femoral tunnel and remained within bone throughout the length of the pin. However, the tip of the drill pins had perforated the lateral cortex of the lateral femoral condyle and became laterally prominent (Figure 5).

Table 1 shows the measurements performed to define the position of the femoral sockets in relation to the posterior and distal articular surfaces of the lateral femoral condyles.

The distance from the starting point of the cross pin sleeves and the femoral epicondyles:

Inclination (A) the starting point for these pins found to be posterior to the level of the lateral epicondyle of the femur with an average entry point of $3.2 \pm 2 \mathrm{~mm}$ for the distal pin and $11.7 \pm 2.3$ (mean $\pm \mathrm{SD}$ ) for the proximal pins (Figure 6).

For pins drilled through inclination (B), the proximal and distal pins were found to perforate the lateral cortex through or anterior to the lateral epicondyle.

On the medial side, inclination C, pins inserted through the medial portal remained through the center of the femoral tunnel. The average inclination of the guide to the posterior femoral condylar line was $41^{\circ}$. When the pins were fully inserted through the guide, penetration of the lateral cortex occurred by both pins. When $7 \mathrm{~mm}$ of the sleeves were lift outside the guide the tip of the drilling pin remained consistently within bone without penetration of the lateral femoral condyle Table 2. Pins inserted through the medial inclination (C) did not harm the articular surface or the medial epicondyle Table 2.

\section{Discussion}

The most clinically relevant findings from this study were: first, the inclination of the guide for double cross pin system alters the position of the pins in the distal femur. An anterior to posterior inclination of the sleeves can lead to penetration of the posterior cortex of the distal femur by the tip of the more distal of the two pins. The second was that if the inclination of the guide was correctly chosen to ensure that the cross pins remain within bone, the entry point for the pins' sleeves will be very close to the attachment of the lateral collateral ligament at the lateral epicondyle. Lastly, a medial entry point for the double cross pins system might provide a safer alternative. However, adjustment of the instruments have to be made to avoid penetration of the lateral cortex of the lateral femoral condyle by the tip of the cross pins.

Anatomic and kinematic studies have shown that anatomic ACL reconstruction can reproduce the function of both bundles of the normal ACL (antero-medial AM and postero-lateral PL) than the older technique that replaced only the AM bundle [2] [8]-[10]. To achieve anatomic insertion of the ACL graft through the natural ACL footprint the medial portal technique of reaming is favored over the trans-tibial method of preparation of the femoral tunnel [2]. Consequently, many surgeons have modified their technique for ACL reconstruction to use the medial portal for preparation of the femoral tunnel. 
Inclination C

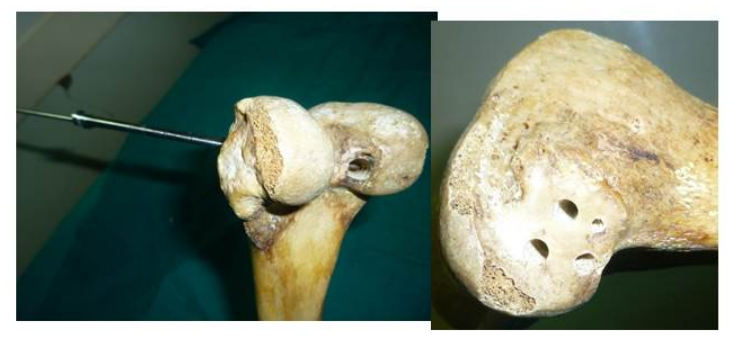

Figure 5. Pins inserted through inclination C achieved central position within the femoral tunnel (left image); however, penetrated the lateral cortex of the lateral femoral condyle (right image).

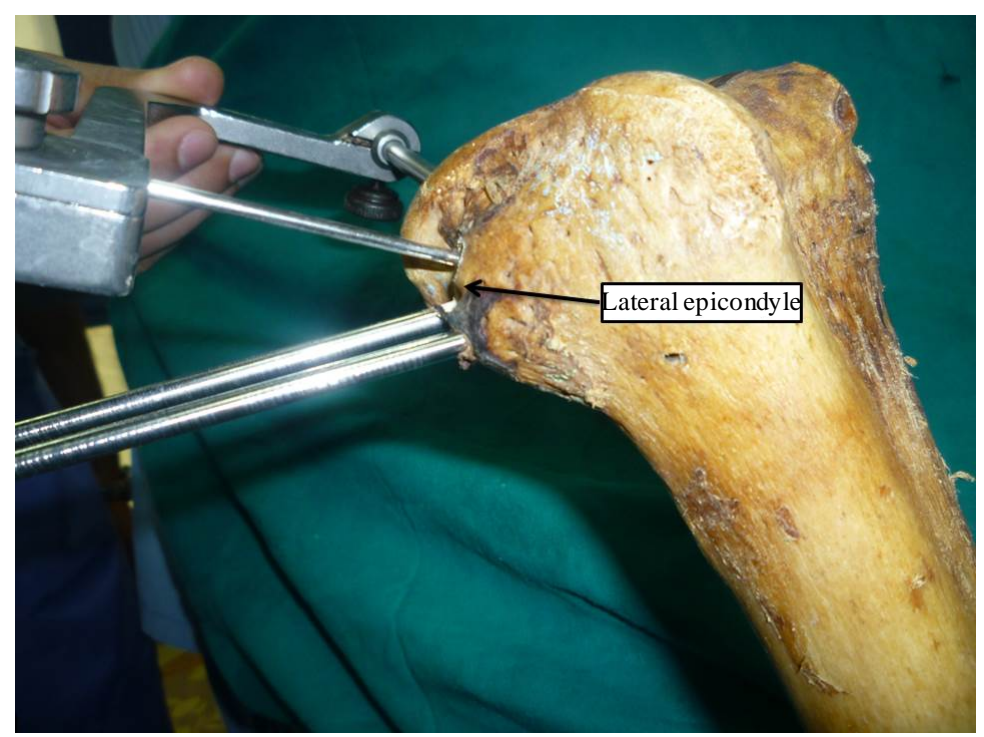

Figure 6. Sleeves of the cross pins inserted through the lateral side were very close to the Lateral epicondyle.

Table 1. This table defines the position of the femoral tunnels in relation to the Distal Articular Cartilage (DAC), Posterior Articular Cartilage (PAC) and the relation between Rigid-Fix sleeves driven through inclination (A) posterior to the Lateral Epicondyle as an anatomic landmark. All distances were measured in mm.

\begin{tabular}{ccccc}
\hline Sample ID & Tunnel to DAC & Tunnel to PAC & $\begin{array}{c}\text { Distal Rigid Fix Pin to lateral } \\
\text { epicondyle }\end{array}$ & $\begin{array}{c}\text { Proximal Rigid Fix Pin to lateral } \\
\text { epicondyle }\end{array}$ \\
\hline R1 & 5 & 2 & 4 & 12 \\
R2 & 7 & 2 & 3 & 11 \\
R3 & 6 & 0 & 7 & 16 \\
R4 & 4 & 2 & 0 & 8 \\
R5 & 8 & 1 & 1 & 13 \\
L1 & 3 & 1 & 3 & 13 \\
L2 & 5 & 2 & 5 & 12 \\
L3 & 4 & 2 & 5 & 13 \\
L4 & 8 & 2 & 2 & 10 \\
L5 & 8 & 3 & 5
\end{tabular}


Table 2. Data collected when the medial entry point (C) was tried regarding Length of sleeve outside the guide, Pins penetrate the lat. Cortex Distance from pins to medial epicondyle and articular surface and Inclination of the guide to the bicondylar axis.

\begin{tabular}{|c|c|c|c|c|c|c|}
\hline \multirow{2}{*}{ Specimen ID } & \multirow{2}{*}{$\begin{array}{l}\text { Length of sleeve } \\
\text { outside the guide }\end{array}$} & \multicolumn{2}{|c|}{$\begin{array}{c}\text { Pins penetrate the lat. Cortex } \\
\text { yes or no }\end{array}$} & \multirow{2}{*}{$\begin{array}{l}\text { Distance from pins to } \\
\text { medial epicondyle }\end{array}$} & \multirow{2}{*}{$\begin{array}{l}\text { Distance from pins } \\
\text { to articular surface }\end{array}$} & \multirow{2}{*}{$\begin{array}{c}\text { Inclination of the } \\
\text { guide to the } \\
\text { bicondylar axis }\end{array}$} \\
\hline & & $\begin{array}{l}\text { Antero-Superior } \\
\text { Pin }\end{array}$ & $\begin{array}{l}\text { Postero-Inferior } \\
\text { Pin }\end{array}$ & & & \\
\hline$L F 2$ & $5 \mathrm{~mm}$ & yes & no & $30 \mathrm{~mm}$ & $6 \mathrm{~mm}$ & $40^{\circ}$ \\
\hline$L F 6$ & $7 \mathrm{~mm}$ & no & no & $32 \mathrm{~mm}$ & $9 \mathrm{~mm}$ & $45^{\circ}$ \\
\hline$L F 7$ & $7 \mathrm{~mm}$ & no & no & $22 \mathrm{~mm}$ & $3 \mathrm{~mm}$ & $40^{\circ}$ \\
\hline$L F 9$ & 0 & yes & yes & $26 \mathrm{~mm}$ & $4 \mathrm{~mm}$ & $50^{\circ}$ \\
\hline$L F 10$ & $7 \mathrm{~mm}$ & no & no & $25 \mathrm{~mm}$ & $13 \mathrm{~mm}$ & $30^{\circ}$ \\
\hline$R F 4$ & $4 \mathrm{~mm}$ & yes & no & $30 \mathrm{~mm}$ & $16 \mathrm{~mm}$ & $45^{\circ}$ \\
\hline$R F 5$ & $6 \mathrm{~mm}$ & no & no & $25 \mathrm{~mm}$ & $7 \mathrm{~mm}$ & $40^{\circ}$ \\
\hline$R F 7$ & $6 \mathrm{~mm}$ & yes & no & $25 \mathrm{~mm}$ & $7 \mathrm{~mm}$ & $45^{\circ}$ \\
\hline$R F 8$ & $7 \mathrm{~mm}$ & no & no & $18 \mathrm{~mm}$ & $16 \mathrm{~mm}$ & $30^{\circ}$ \\
\hline$R F 9$ & $7 \mathrm{~mm}$ & no & no & $30 \mathrm{~mm}$ & $10 \mathrm{~mm}$ & $50^{\circ}$ \\
\hline Average & 5.6 & - & - & 26.3 & 9.1 & 41.5 \\
\hline$S D$ & 2.2 & - & - & 4.3 & 4.6 & 7.1 \\
\hline
\end{tabular}

Preparation of the femoral tunnel through the medial portal results in change of the inclination and relatively shorter femoral tunnels when compared to the trans-tibial technique. Therefore, choosing a method for securing the ACL graft on the femoral side has to take these points into consideration.

Methods of fixing the ACL graft on the femoral side that rely on cortical suspension usually occupy part of the femoral tunnel $(15-20 \mathrm{~mm})$ with a suspension loop. If the femoral tunnel is already shortened with the medial portal preparation technique, presence of suspension loop at the far part of the femoral tunnel may reduce the contact length between the ACL graft and the host bone at the femoral side. From that prospect the double cross pin method of fixation would give the advantage of longer area of contact between the ACL graft and the whole length of the femoral tunnel. The clinical impact of these changes is not yet clear.

The double cross pins (Rigid-Fix, MITEK, Johnson \& Johnson) method of fixation that secures the ACL graft at the femoral side had originally been designed to work through the trans-tibial technique. It is essential to assess the suitability of this fixation method to the current trend of using the medial portal for anatomic preparation of the ACL graft.

Recent cadaveric study evaluated the safety of transverse pin (Stryker system) originally designed for transtibial technique in anatomic single bundle ACL reconstruction. The study revealed high risk of injury to the lateral collateral ligament and even the popliteus tendon [5].

The cross pin system (Rigid-Fix) has a smaller diameter but still introduced very close to the attachment of the lateral collateral ligament origin at the lateral epicondyle. Therefore, medial entry point may prove to be a safer approach for insertion of the pins.

This study has some limitations. The study was conducted on cadaveric bone. Full cadaveric trial on a larger number of samples would be necessary.

\section{Conclusion}

The bio-absorbable double cross pins (Rigid-Fix, MITEK, J\&J) that was designed for trans-tibial technique should carefully be used in anatomic single bundle ACL reconstruction. The risk of penetration of the posterior cortex of the femur suggests alteration of the technique to achieve safe insertion of the pins.

\section{Conflict of Interest}

Conflict of interest to all authors: None. 


\section{Ethical Committee Approval}

This study was approved by the local research committee and follows the laws of cadaveric and clinical research at our institute.

\section{References}

[1] Van Eck, C., Working, Z. and Fu, F. (2011) Current Concepts in Anatomic Single- and Double-Bundle Anterior Cruciate Ligament Reconstruction. The Physician and Sportsmedicine, 39, 140-148.

[2] Bedi, A., Musahl, V., Steuber, V., Kendoff, D., Choi, D., Allen, A.A., Pearle, A.D. and Altchek, D.W. (2010) Transtibial versus Anteromedial Portal Reaming in Anterior Cruciate Ligament Reconstruction: An Anatomic and Biomechanical Evaluation of Surgical Technique. Arthroscopy, 27, 380-390. http://dx.doi.org/10.1016/j.arthro.2010.07.018

[3] Baumfeld, J.A., Diduch, D.R., Rubino, L.J., Hart, J.A., Miller, M.D., Barr, M.S., et al. (2008) Tunnel Widening Following Anterior Cruciate Ligament Reconstruction Using Hamstring Autograft: A Comparison between Double CrossPin and Suspensory Graft Fixation. Knee Surgery, Sports Traumatology, Arthroscopy, 16, 1108-1113. http://dx.doi.org/10.1007/s00167-008-0606-y

[4] Zantop, T., Weimann, A., Wolle, K., Musahl, V., Langer, M. and Petersen, W. (2007) Initial and 6 Weeks Postoperative Structural Properties of Soft Tissue Anterior Cruciate Ligament Reconstructions with Cross-Pin or Interference Screw Fixation: An in Vivo Study in Sheep. Arthroscopy, 23, 14-20. http://dx.doi.org/10.1016/j.arthro.2006.10.007

[5] Gelber, P.E., Reina, F., Torres, R., Pelfort, X., Tey, M. and Monllau, J.C. (2010) Anatomic Single-Bundle Anterior Cruciate Ligament Reconstruction from the Anteromedial Portal: Evaluation of Transverse Femoral Fixation in a Cadaveric Model. Arthroscopy, 26, 651-657. http://dx.doi.org/10.1016/j.arthro.2009.09.020

[6] Chang, C.B., Yoo, J.H., Chung, B.J., Seong, S.C. and Kim, T.K. (2010) Oblique Femoral Tunnel Placement Can Increase Risks of Short Femoral Tunnel and Cross-Pin Protrusion in Anterior Cruciate Ligament Reconstruction. The American Journal of Sports Medicine, 38, 1237-1245. http://dx.doi.org/10.1177/0363546509357608

[7] Van Eck, C.F., Lesniak, B.P., Schreiber, V.M. and Fu, F.H. (2010) Anatomic Single- and Double-Bundle Anterior Cruciate Ligament Reconstruction Flowchart. Arthroscopy, 26, 258-268. http://dx.doi.org/10.1016/j.arthro.2009.07.027

[8] Bedi, A., Musahl, V., Steuber, V., Kendoff, D., Choi, D., Allen, A.A., et al. (2011) Transtibial versus Anteromedial Portal Reaming in anterior Cruciate Ligament Reconstruction: An Anatomic and Biomechanical Evaluation of Surgical Technique. Arthroscopy, 27, 380-390. http://dx.doi.org/10.1016/j.arthro.2010.07.018

[9] Kondo, E., Merican, A.M., Yasuda, K. and Amis, A.A. (2011) Biomechanical Comparison of Anatomic Double-Bundle, Anatomic Single-Bundle, and Nonanatomic Single-Bundle Anterior Cruciate Ligament Reconstructions. The American Journal of Sports Medicine, 39, 279-288. http://dx.doi.org/10.1177/0363546510392350

[10] Noyes, F.R. and Barber-Westin, S.D. (2011) Anterior Cruciate Ligament Graft Placement Recommendations and Bone-Patellar Tendon-Bone Graft Indications to Restore Knee Stability. Instructional Course Lectures, 60, 499-521. 
Scientific Research Publishing (SCIRP) is one of the largest Open Access journal publishers. It is currently publishing more than 200 open access, online, peer-reviewed journals covering a wide range of academic disciplines. SCIRP serves the worldwide academic communities and contributes to the progress and application of science with its publication.

Other selected journals from SCIRP are listed as below. Submit your manuscript to us via either submit@scirp.org or Online Submission Portal.
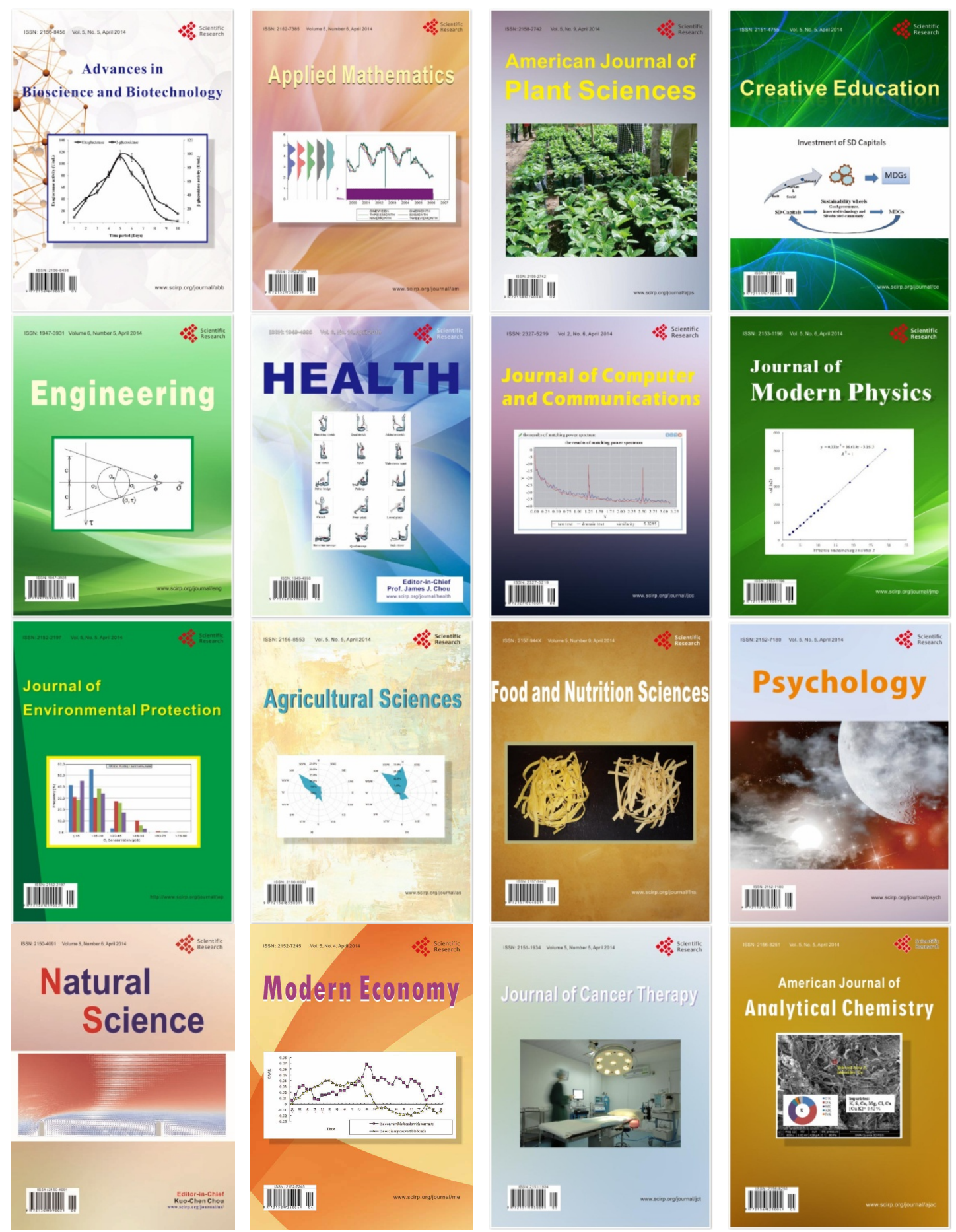